\title{
Crimean-Congo hemorrhagic fever in Europe: current situation calls for preparedness
}

H C Maltezou (helen-maltezou@ath.forthnet.gr) ${ }^{1}$, L Andonova², R Andraghetti ${ }^{3}$, M Bouloy $^{4}, 0$ Ergonul5, F Jongejan ${ }^{6}$,

N Kalvatchev ${ }^{7}$, S Nichol ${ }^{8}$, M Niedrig ${ }^{9}$, A Platonov ${ }^{10}$, G Thomson ${ }^{11}$, K Leitmeyer $^{12}$, H Zeller $^{12}$

1. Hellenic Center for Diseases Control and Prevention, Athens, Greece

2. Medical University, Sofia, Bulgaria

3. World Health Organization, Copenhagen, Denmark

4. Institut Pasteur, Paris, France

5. Marmara University, Istanbul, Turkey

6. Utrecht Centre for Tick-borne Diseases, Utrecht University, Utrecht, the Netherlands

7. National Centre of Infectious and Parasitic Diseases, Sofia, Bulgaria

8. Centers of Disease Control and Prevention, Atlanta, United States

9. Robert Koch Institute, Berlin, Germany

10. Central Research Institute of Epidemiology, Moscow, Russian Federation

11. Health Protection Agency, London, United Kingdom

12. European Centre for Disease Control and Prevention, Stockholm, Sweden

Citation style for this article:

Citation style for this article: Maltezou HC, Andonova L, Andraghetti R, Bouloy M, Ergonul O, Jongejan F, Kalvatchev N, Nichol S, Niedrig M, Platonov A, Thomson G, Leitmeyer K, Zeller H. Crimean-Congo hemorrhagic fever in Europe: current situation calls for preparedness. Euro Surveill. 2010;15(10):pii=19504. Available online: http://www.eurosurveillance.org/ViewArticle.aspx?Articleld $=19504$

This article has been published on 11 March 2010

During the last decade Crimean-Congo hemorrhagic fever (CCHF) emerged and/or re-emerged in several Balkan countries, Turkey, southwestern regions of the Russian Federation, and the Ukraine, with considerable high fatality rates. Reasons for re-emergence of CCHF include climate and anthropogenic factors such as changes in land use, agricultural practices or hunting activities, movement of livestock that may influence host-tick-virus dynamics. In order to be able to design prevention and control measures targeted at the disease, mapping of endemic areas and risk assessment for CCHF in Europe should be completed. Furthermore, areas at risk for further CCHF expansion should be identified and human, vector and animal surveillance be strengthened.

\section{Introduction}

Crimean-Congo hemorrhagic fever (CCHF) is an acute, highly-contagious viral zoonosis transmitted to humans mainly by ticks of the genus Hyalomma, but also through direct contact with blood or tissues of viraemic hosts. In humans CCHF typically presents with high fever of sudden onset, malaise, severe headache and gastrointestinal symptoms. Prominent hemorrhages may occur in late stages of the disease with published fatality rates ranging from $10 \%$ to $50 \%[1,2]$. The disease is endemic in parts of Africa, Asia, the Middle East and eastern Europe. Main animal hosts include a number of domestic animals such as cattle, sheep, goats, and hares. CCHF has the potential to cause community and nosocomial outbreaks. Due to the high case fatality rates and difficulties in treatment, prevention, and control, CCHF is a disease which should be notified immediately to public health authorities in the European Union (EU). CCHF virus is also in the list of agents for which the Revised International Health Regulations of 2005 call for implementation of the decision algorithm for risk assessment and possible notification to the World Health Organization (WHO) [3].

In Europe, CCHF is currently only endemic in Bulgaria, however during the last decade an increased number of CCHF cases and outbreaks have been recorded in other countries in the region such as Albania, Kosovo, Turkey, and the Ukraine as well as south-western regions of the Russian Federation [4-9]. In June 2008, the first case was registered in Greece [10]. In response to this situation, the European Centre for Disease Prevention and Control (ECDC) invited a group of CCHF experts to review the situation of CCHF in Europe and to consult on interventions necessary to strengthen preparedness and response at the European level [11]. This article provides an update on the current situation of CCHF in Europe, and emphasises existing prevention and control capacities within the EU. Aspects relevant to strengthen preparedness for CCHF are also discussed.

\section{CCHF situation in Europe}

CCHF is endemic in Bulgaria since the 1950's, when a large outbreak occurred from 1954 to 1955 with 487 notified cases mainly in the Shumen area in north-east Bulgaria. In total, 1,568 CCHF cases were notified in Bulgaria from 1953 to 2008, with an overall case fatality rate of $17 \%$ [4]. Endemic areas are confined to the vicinity of Shumen, Razgrad, Veliko Tarnovo, Plovdiv, Pazardjik, Haskovo, Kardjali, and Bourgas, however in April 2008 a cluster of six probable cases occurred in Gotse Delchev in the south-western province 
Blagoevgrad near the border with Greece, an area considered of low CCHF endemicity until recently [5]. During the last decade, CCHF outbreaks have also been noted in Albania in 2001 and 2003, and in Kosovo in $2001[6,7]$.

In Turkey, the first symptomatic human CCHF cases were noted in 2002, however, serologic evidence of enzootic CCHF virus circulation as well as limited evidence of CCHF infections among humans (2.4\% among 1,100 tested humans) has been found since the 1970's [4]. Starting in 2003, Turkey has experienced an expanding outbreak with increasing numbers of notified cases and associated fatalities (2002: 17/0; 2003: 133/6; 2004: 249/13; 2005: 266/13; 2006: 438/27; 2007: 713/33; 2008: 1315/63; 2009: 1300/62) [4,12]. Overall, there are more than 4,400 recorded laboratory confirmed CCHF cases in this country, mainly among residents in rural areas in north-central and northeast Anatolia $[4,8,12]$. Within the CCHF endemic areas, there are hyperendemic areas where one out of every five residents and one out of every two residents with a history of tick bite has antibodies against CCHF virus [13]. A predictive map model using satellite-based climate data and high-resolution vegetation images from Turkey from 2003 to 2006 revealed that areas with higher CCHF reporting were significantly associated with zones of high climate suitability for Hyalomma ticks and high rate of fragmentation of agricultural land [13].

In Greece, a serosurvey conducted between 1981 and 1988 among 3,388 rural residents from across the country showed $1 \%$ seroprevalence rate against $\mathrm{CCHF}$ virus [4]. More than 400 cases with a CCHF compatible clinical syndrome have tested negative for CCHF virus in this country since 1982, therefore, the seroprevalence rate of $1 \%$ was attributed to the non-pathogenic AP-92 strain and not to the pathogenic Balkan CCHF virus strain. A number of the cases tested for CCHF were finally diagnosed as hemorrhagic fever with renal syndrome (HFRS), leptospirosis and ricketsial infections. Other diagnoses were meningococcal meningitis and unspecified bacterial sepsis. The first CCHF case was recorded in June 2008 in a woman with a tick bite working in agriculture near the city of Komotini in north-eastern Greece [10]. This town is situated within a few kilometres distance from there where the Bulgarian cluster occurred in 2008 [5]. A seroepidemiological study for CCHF virus among local population and animals are underway in northern Greece.

After nearly 27 years without any human cases, CCHF re-emerged in the south-western regions of the Russian Federation in 1999. Outbreaks have been reported in Astrakhan, Rostov and Volgograd Provinces, Krasnodar and Stavropol Territories, Kalmykia, Dagestan and Ingushetia Republics. Between 2000 and 2009 more than 1,300 clinical cases were diagnosed in the Russian Federation with an overall fatality rate of $3.2 \%$ for the period from 2002-2007 [4]. Most cases occurred among residents of rural areas in the Southern Federal Distinct. The largest number of cases was registered in Stavropol Territory, Kalmykia Republic and Rostov Province, where the mean annual CCHF incidence rate was $1.7,10.1$, and 0.7 cases per 100,000 population, respectively. During 2008 alone, the incidence in Stavropol Territory increased by 1.3 times, and was the highest recorded in this region during the last decade $[4,9,14]$. In 2009, CCHF cases were also reported from Georgia, Kazakhstan, Tajikistan, Iran, and Pakistan [15].

CCHF emergence and/or re-emergence in south-eastern Europe and neighboring countries is attributed to climate and ecologic changes and anthropogenic factors such as changes in land use, agricultural practices, hunting activities, and movement of livestock, that may have an impact on ticks and hosts and accordingly on CCHF epidemiology $[1,2]$. The geographic distribution of CCHF coincides with that of Hyalomma ticks. $H$. marginatum, the main CCHF virus vector in Europe, is found in Albania, Bulgaria, Cyprus, France, Greece, Italy, Kosovo, Moldavia, Portugal, Romania, Russia, Serbia, Spain, Turkey, and the Ukraine. In 2006 it was detected for the first time in the Netherlands and in southern Germany $[16,17]$. Given the wide distribution of its vector, the numerous animals that can serve as hosts, and the favorable climate and ecologic conditions in several European countries bordering the Mediterranean Sea, it is possible that the occurrence of CCHF will expand in the future. A model that studied various climate scenarios on the habitat areas of different ticks, showed that a rise in temperature and a decrease in rainfall in the Mediterranean region will result in a sharp increase in the suitable habitat areas for $H$. marginatum and its expansion towards the north, with the highest impact noted at the margins of its current geographic range [18].

\section{Current prevention and control in Europe}

Several elements relating to laboratory diagnosis, surveillance and therapy of CCHF should be addressed in order to increase preparedness capacity in Europe and to design appropriate prevention and control measures.

\section{Laboratory diagnosis}

In 2008 there were 20 laboratories with diagnostic capacities for CCHF virus in Europe: 14 in EU Member States, eight in the endemic regions of the Russian Federation, and one in Turkey. Most of them used immunofluorescence assays (IFA), ELISA, and/or molecular methods to diagnose CCHF whereas eight among them were also able to isolate CCHF virus [11], a BSL-4 containment agent. Limitations for diagnosing CCHF concern both the limited diagnostic capacities in several endemic areas as well as difficulties in the international transfer of samples for logistic and economic reasons. However, rapid and easy tests are needed to guide initial therapeutic decisions for the patient. 


\section{Surveillance}

Currently, there are no standardised case definitions for CCHF notification and contact tracing within European countries [19]. Recent cases of nosocomial acquisition of CCHF in health care workers were well documented $[6,8,20]$. These cases underline the need for educating health-care workers about the modes of getting infected with CCHF virus and for strict implementation of infection control measures within health-care facilities, and the importance of providing adequate resources to do so $[1,2]$.

\section{Therapy}

The World Health Organization (WHO) recommends ribavirin for the treatment of CCHF cases [21,22]. Ribavirin appears to be more effective when introduced early in the course of illness [23]. Evidence of its efficacy is based on in vitro data and on limited observations in humans [24-26]. Randomised controlled trials have not been conducted so far, and ethical issues concerning the use of a control group remain a major obstacle for this [27]. Severity of infection, duration of illness prior to initiation of therapy, and route of administration may impact the clinical outcome of CCHF cases. On individual country level, recommendations for treatment of CCHF cases with ribavirin existed in 2008 in Turkey, Russia, Bulgaria, and Greece. In Bulgaria, in addition, specific hyperimmune globulin collected from convalescent CCHF cases is used for prophylaxis and treatment and an inactivated suckling mouse brain vaccine is in use since the 1970's for high-risk groups living in CCHF endemic regions [28]. There is no vaccine against CCHF licensed in any other EU Member State.

\section{Conclusions}

CCHF is a disease with a high fatality rate and the potential to cause outbreaks. The vector for CCHF, the Hyalomma tick is present in southeastern and southern Europe. Climate factors may contribute to a further spread of the vector and to a consecutive extension of the geographic range of $\mathrm{CCHF}$, which may further expand to European countries bordering the Mediterranean Sea, with the highest risk in neighbouring areas with already established endemicity. This highlights the need for strengthening human, vector, and veterinary surveillance, especially in areas where CCHF is expected to occur in the future. Together with the implementation of standardised case definitions for CCHF this will allow an estimate of the CCHF burden and of epidemiologic trends in various areas and countries. Guidance for contact tracing and the establishment of early detection and response systems will allow prompt interventions at patient, community, and hospital level. To enable early detection, laboratory capacities are crucial to rapidly confirm the suspected clinical diagnosis and besides being available, tests need to be reliable and affordable. Overall, laboratory capacities for CCHF should increase. Considering the high case fatality rate of CCHF, development of a vaccine and new drugs against CCHF are of major importance. Ribavirin efficacy should be assessed through well-designed clinical protocols and in endemic areas general public and health-care workers should be aware about modes of CCHF transmission and prophylactic measures. Climate and environmental factors and human behavior that may influence CCHF epidemiology and spread should be further studied. Mapping of endemic areas and risk assessment for CCHF in Europe should be completed and areas at risk for CCHF expansion should be identified and finally, appropriate tick-control strategies including public education should be implemented. All these measures should be undertaken as part of a multidisciplinary collaboration at interregional and international level and link with initiatives such as the International network for capacity building for the control of emerging viral vectorborne zoonotic diseases: ARBO-ZOONET [29].

In accordance with an ECDC-initiated assessment on the importance of vector-borne diseases in 2008 , CCHF has been identified as a priority disease for the EU [12]. In order to strengthen preparedness and response for CCHF and build capacity for its prevention and control, it is necessary to identify relevant gaps and work in an integrated fashion.

\section{References}

1. Vorou R, Pierroutsakos IN, Maltezou HC. Crimean-Congo hemorrhagic fever. Curr Opin Infect Dis. 2007;20(5):495-500.

2. Ergönül 0 . Crimean-Congo haemorrhagic fever. Lancet Infect Dis. 2006;6(4):203-14.

3. World Health Organization (WHO). [Internet]. International Health Regulations (2005). 2nd ed. Geneva, WHO 2008. Available from: http://whqlibdoc.who.int/ publications/2008/9789241580410_eng.pdf

4. World Health Organization Regional Office for Europe (WHO). Epidemiology for Crimean-Congo haemorrhagic fever virus: Turkey, Russian Federation, Bulgaria, Greece, Albania, Kosovo. Available from: www.euro.who.int/surveillance/ outbreaks/20080806_1

5. Kunchev A, Kojouharova M. Probable cases of Crimean-Congohaemorrhagic fever in Bulgaria: a preliminary report. Euro Surveill. 2008;13(17). pii=18845. Available from: http://www. eurosurveillance.org/ViewArticle.aspx?Articleld=18845

6. Papa A, Bino S, Llagami A, Brahinaj B, Papadimitriou E, Pavlidou V, et al. Crimean-Congo hemorrhagic fever in Albania, 2001. Eur J Clin Microbiol Infect Dis. 2002;21(8):603-6.

7. Papa A, Bozovi B, Pavlidou V, Papadimitriou E, Pelemis M, Antoniadis A. Genetic detection and isolation of crimeancongo hemorrhagic fever virus, Kosovo, Yugoslavia. Emerg Infect Dis. 2002;8(8):852-4.

8. Yilmaz GR, Buzgan T, Irmak H, Safran A, Uzun R, Cevik MA, et al. The epidemiology of Crimean-Congo hemorrhagic fever in Turkey, 2002-2007. Int J Infect Dis. 2009;13(3):380-6.

9. Federal Service for Surveillance on Consumer Rights Protection and Wellbeing, the Russian Federation. [Internet] On improvement preventive measures against Crimean-Congo haemorrhagic fever in Southern Federal District. Letter of 11.03.2009. Available from: http://www.rospotrebnadzor.ru/ documents/letters/2410/ [Russian].

10. Papa A, Maltezou HC, Tsiodras S, Dalla VG, Papadimitriou T, Pierroutsakos IN, et al. A case of Crimean-Congo haemorrhagic fever in Greece, June 2008. Euro Surveill. 2008;13(33). pii:18952. Available from: http://www. eurosurveillance.org/ViewArticle.aspx?Articleld $=18952$

11. European Centre for Disease Prevention and Control (ECDC). Meeting report: Consultation on Crimean-Congo haemorrhagic fever prevention and control. Stockholm, September 2008. Available from: http://ecdc.europa.eu/en/publications/ Publications/0809_MER_Crimean_Congo_Haemorragic_Fever Prevention_and_Control.pdf

12. Turkish Ministry of Health. [Internet]. Tarihli Aciklama, September 25, 2009. Available from: http://www.saglik.gov. tr/KKKA/BelgeGoster.aspx?F6E10F8892433CFFA91171E62FoFF1 532A030B47EF2AC66C [Turkish]. 
13. Estrada-Peña A, Zatansever Z, Gargili A, Aktas M, Uzun R, Ergonul 0, et al. Modeling the spatial distribution of crimeancongo hemorrhagic fever outbreaks in Turkey. Vector Borne Zoonotic Dis. 2007;7(4):667-78.

14. Maletskaya OV, Beyer AP, Agapitov DS, Kharchenko TV, Taran AV, Taran TV, et al. Epidemic situation on Kongo-Crimean hemorrhagic fever in South Federal District of Russia. Zh Mikrobiol Epidemiol Immunobiol 2009;(6):51-4 [In Russian].

15. World Organisation for Animal Health (OIE), World Health Organization (WHO), Food and Agriculture Organization of the United Nations (FAO). [Internet]. Global Early Warning and Response System for Major Animal Diseases, including Zoonoses. November 26, 2009. Available from: http://www. glews.net/index.php?option=com_content\&view=article\&id= 85:crimean-congo-hemorrhagic-fever-cchf\&catid $=64$ : diseasepriority-list

16. Nijhof AM, Bodaan C, Postigo M, Nieuwenhuijs H, Opsteegh M, Franssen L, et al. Ticks and associated pathogens collected from domestic animals in the Netherlands. Vector Borne Zoonotic Dis. 2007;7(4):585-95.

17. Kampen H, Poltz W, Hartelt K, Wolfel R, Faulde M. Detection of a questing Hyalomma marginatum marginatum adult female (Acari, Ixodidae) in southern Germany. Exp Appl Acarol. 2007;43(3):227-31.

18. Estrada-Peña A, Venzal JM. Climate niches of tick species in the Mediterranean region: modeling of occurrence data, distributional constraints, and impact of climate change. I Med Entomol. 2007;44(6):1130-8.

19. Maltezou HC, Papa A, Tsiodras S, Dalla V, Maltezos E, Antoniadis A. Crimean-Congo hemorrhagic fever in Greece: a public health perspective. Int J Infect Dis. 2009;13(6):713-6.

20. Harxhi A, Pilaca A, Delia Z, Pano K, Rezza G. Crimean-Congo hemorrhagic fever: a case of nosocomial transmission. Infection. 2005;33(4):295-6.

21. World Health Organization (WHO). WHO Model List of Essential Drugs, 2007. Available from: http://www.who.int/entity/ medicines/publications/08_ENGLISH_indexFINAL_EML15.pdf

22. World Health Organization (WHO). WHO Model Formulary 2008. Available from: http://www.who.int/entity/selection medicines/list/WMF2008.pdf

23. Tasdelen Fisgin N, Ergonul O, Doganci L, Tulek N. The role of ribavirin in the therapy of Crimean-Congo hemorrhagic fever: early use is promising. Eur J Clin Microbiol Infect Dis. 2009;28(8):929-33.

24. Paragas J, Whitehouse CA, Endy TP, Bray M. A simple assay for determining antiviral activity against Crimean-Congo hemorrhagic fever virus. Antiviral Res. 2004;62(1):21-5.

25. Watts DM, Ussery MA, Nash D, Peters CJ. Inhibition of CrimeanCongo hemorrhagic fever viral infectivity yields in vitro by ribavirin. Am J Trop Med Hyg. 1989;41(5):581-5.

26. Mardani M, Jahromi MK, Naeni KH, Zeinali M. The efficacy of oral ribavirin in the treatment of crimean-congo hemorrhagic fever in Iran. Clin Infect Dis. 2003;36(12):1613-8.

27. Ergonul 0. Treatment of Crimean-Congo hemorrhagic fever. Antivir Res. 2008;78(1):125-31

28. Papa A, Christova I, Papadimitriou E, Antoniadis A. CrimeanCongo hemorrhagic fever in Bulgaria. Emerg Infect Dis. 2004;10(8):1465-7.

29. Ahmed J, Bouloy M, Ergonul O, Fooks A, Paweska J, Chevalier V, et al. International network for capacity building for the control of emerging viral vector-borne zoonotic diseases: ARBOZOONET. Euro Surveill. 2009;14(12). pii: 19160. http://www. eurosurveillance.org/ViewArticle.aspx?Articleld=19160 